\title{
Improved singular spectrum analysis for time series with missing data
}

\author{
Y. Shen ${ }^{1}$, F. Peng ${ }^{1,2}$, and B. $\mathbf{L i}^{1}$ \\ ${ }^{1}$ College of Surveying and Geo-informatics, Tongji University, Shanghai, China \\ ${ }^{2}$ Center for Spatial Information Science and Sustainable Development, Shanghai, China \\ Correspondence to: Y. Shen (yzshen@tongji.edu.cn)
}

Received: 12 October 2014 - Published in Nonlin. Processes Geophys. Discuss.: 21 December 2014

Revised: 15 May 2015 - Accepted: 20 May 2015 - Published: 10 July 2015

\begin{abstract}
Singular spectrum analysis (SSA) is a powerful technique for time series analysis. Based on the property that the original time series can be reproduced from its principal components, this contribution develops an improved SSA (ISSA) for processing the incomplete time series and the modified SSA (SSAM) of Schoellhamer (2001) is its special case. The approach is evaluated with the synthetic and real incomplete time series data of suspended-sediment concentration from San Francisco Bay. The result from the synthetic time series with missing data shows that the relative errors of the principal components reconstructed by ISSA are much smaller than those reconstructed by SSAM. Moreover, when the percentage of the missing data over the whole time series reaches $60 \%$, the improvements of relative errors are up to $19.64,41.34,23.27$ and $50.30 \%$ for the first four principal components, respectively. Both the mean absolute error and mean root mean squared error of the reconstructed time series by ISSA are also smaller than those by SSAM. The respective improvements are 34.45 and $33.91 \%$ when the missing data accounts for $60 \%$. The results from real incomplete time series also show that the standard deviation (SD) derived by ISSA is $12.27 \mathrm{mg} \mathrm{L}^{-1}$, smaller than the $13.48 \mathrm{mg} \mathrm{L}^{-1} \mathrm{de}-$ rived by SSAM.
\end{abstract}

\section{Introduction}

Singular spectrum analysis (SSA) introduced by Broomhead and King (1986) for studying dynamical systems is a powerful toolkit for extracting short, noisy and chaotic signals (Vautard et al., 1992). SSA first transfers a time series into a trajectory matrix, and carries out the principal component analysis to pick out the dominant components of the trajectory matrix. Based on these dominant components, the time series is reconstructed. Therefore the reconstructed time series improves the signal-to-noise ratio and reveals the characteristics of the original time series. SSA has been widely used in geosciences to analyse a variety of time series, such as the stream flow and sea-surface temperature (Robertson and Mechoso, 1998; Kondrashov and Ghil, 2006), the seismic tomography (Oropeza and Sacchi, 2011) and the monthly gravity field (Zotova and Shum, 2010). Schoellhamer (2001) developed a modified SSA for time series with missing data (SSAM), which was successfully applied to analyse the time series of suspended-sediment concentration (SSC) in San Francisco Bay (Schoellhamer, 2002). This SSAM approach does not need to fill missing data. Instead, it computes each principal component (PC) with observed data and a scale factor related to the number of missing data. Shen et al. (2014) developed a new principal component analysis approach for extracting common mode errors from the time series with missing data of a regional station network. The other kind of SSA approach process the time series with missing data by filling the data gaps recursively or iteratively, such as the "Caterpillar" SSA method (Golyandina and Osipov, 2007), the imputation method (Rodrigues and Carvalho, 2013) or the iterative method (Kondrashov and Ghil, 2006).

This paper is motivated by Schoellhamer (2001) and Shen et al. (2014) and develops an improved SSA (ISSA) approach. In our ISSA, the lagged correlation matrix is computed in the same way as by Schoellhamer (2001) - the PCs are directly computed with both the eigenvalues and eigenvectors of the lagged correlation matrix. However, the PCs in Schoellhamer (2001) were calculated with the eigenvec- 
tors and a scale factor to compensate for the missing value. Moreover, we do not need to fill in the missing data recursively and iteratively as in Golyandina and Osipov (2007). The rest of this paper is organized as follows: the improvement of SSA for time series with missing data follows in Sect. 2, synthetic and real numerical examples are presented in Sects. 3 and 4 respectively, and then conclusions are given in Sect. 5.

\section{Improved singular spectrum analysis for time series with missing data}

For a stationary time series $x_{i}(1 \leq i \leq N)$, we can construct an $L \times(N-L+1)$ trajectory matrix with a window size $L$. Its Toeplitz lagged correlation matrix $\mathbf{C}$ is formulated by

$\mathbf{C}=\left[\begin{array}{cccc}c(0) & c(1) & \cdots & c(L-1) \\ c(1) & c(0) & \ddots & \vdots \\ \vdots & \vdots & \ddots & c(1) \\ c(L-1) & \cdots & \cdots & c(0)\end{array}\right]$

Each element $c(j)$ is computed by

$c(j)=\frac{1}{N-j} \sum_{i=1}^{N-j} x_{i} x_{i+j} \quad j=0,1,2, \ldots, L-1$.

For matrix $\mathbf{C}$, we can compute its eigenvalues $\lambda_{k}$ and the corresponding eigenvectors $\boldsymbol{v}_{k}$ in descending order of $\lambda_{k}$ $(1 \leq k \leq L)$. Then the $i$ th element of the $k$ th principal component (PC) $\boldsymbol{a}_{k}$ is computed by

$a_{k, i}=\sum_{j=1}^{L} x_{i+j-1} v_{j, k} \quad 1 \leq i \leq N-L+1$,

where $v_{j, k}$ is the $j$ th element of $\boldsymbol{v}_{k}$. We compute the $k$ th reconstructed components (RCs) of the time series with the $k$ th PC as (Vautard et al., 1992)

$x_{i}^{k}= \begin{cases}\frac{1}{i} \sum_{j=1}^{i} a_{k, i-j+1} v_{j, k} & 1 \leq i \leq L-1 \\ \frac{1}{L} \sum_{j=1}^{L} a_{k, i-j+1} v_{j, k} & L \leq i \leq N-L+1 \\ \frac{1}{N-i+1} \sum_{j=i-N+L}^{L} a_{k, i-j+1} v_{j, k} & N-L+2 \leq i \leq N .\end{cases}$

Since $\lambda_{k}$, the variance of the $k$ th $\mathrm{RC}$, is sorted in descending order, the first several RCs contain most of the signals of the time series, while the remaining RCs contain mainly the noises of time series. Thus the original time series is reconstructed with the first several RCs.

The SSAM approach developed by Schoellhamer (2001) computes the elements $c(j)$ of the lagged correlation matrix by
$c(j)=\frac{1}{N_{j}} \sum_{i \leq N-j} x_{i} x_{i+j} \quad j=0,1,2, \ldots, L-1$,

where both $x_{i}$ and $x_{i+j}$ must be observed rather than missed, and $N_{j}$ is the number of the products of $x_{i}$ and $x_{i+j}$ within the sample index $i \leq N-j$. Then we compute the eigenvalues and eigenvectors from the lagged correlation matrix $\mathbf{C}$. The PCs are also calculated with observed data:

$a_{k, i}=\frac{L}{L_{i}} \sum_{1 \leq j \leq L} x_{i+j-1} v_{j, k} \quad 1 \leq i \leq N-L+1$,

where $L_{i}$ is the number of observed data within the sample index from $i$ to $i+L-1$. The reconstruction procedure of time series from PCs is the same as SSA. The scale factor $L / L_{i}$ is used to compensate for the missing value.

In order to derive the expression of computing PCs for the time series with missing data, Eq. (3) is reformulated as

$a_{k, i}=\sum_{i+j-1 \in S_{i}} x_{i+j-1} v_{j, k}+\sum_{i+j-1 \in \bar{S}_{i}} x_{i+j-1} v_{j, k}$,

where $1 \leq i \leq N-L+1$, and $S_{i}$ and $\bar{S}_{i}$ are the index sets of sampling data and missing data respectively within the integer interval $[i, i+L-1]$, i.e. $S_{i} \cap \bar{S}_{i}=0$ and $S_{i} \cup \bar{S}_{i}=[i$, $i+L-1]$. If PCs are available, we can reproduce the missing values. Therefore, the missing values in Eq. (7) can be substituted with PCs as

$x_{i+j-1}=\sum_{m=1}^{L} a_{m, i} v_{j, m}$.

Substituting Eq. (8) into the second term of the right-hand side of Eq. (7) yields

$$
\begin{aligned}
\left(1-\sum_{i+j-1 \in \bar{S}_{i}} v_{j, k}^{2}\right) a_{k, i} & -\sum_{i+j-1 \in \bar{S}_{i}} \sum_{m=1, m \neq k}^{L} v_{j, m} v_{j, k} a_{m, i} \\
& =\sum_{i+j-1 \in S_{i}} x_{i+j-1} v_{j, k} .
\end{aligned}
$$

Collecting all equations of Eq. (9) for $k=1,2, \ldots, L$, we have

$G_{i} \xi_{i}=\boldsymbol{y}_{i}$,

where

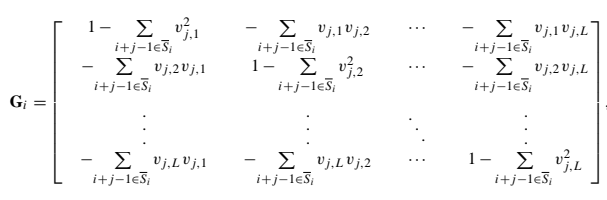


$\xi_{i}=\left[\begin{array}{c}a_{1, i} \\ a_{2, i} \\ \vdots \\ a_{L, i}\end{array}\right], \boldsymbol{y}_{i}=\left[\begin{array}{c}\sum_{i+j-1 \in S_{i}} x_{i+j-1} v_{j, 1} \\ \sum_{i+j-1 \in S_{i}} x_{i+j-1} v_{j, 2} \\ \vdots \\ \sum_{i+j-1 \in S_{i}} x_{i+j-1} v_{j, L}\end{array}\right]$.

Since $\mathbf{G}_{i}$ is a symmetric and rank-deficient matrix with the number of rank deficiency equaling the number of missing data within the interval $\left[x_{i}, x_{i+L-1}\right]$, the PCs $a_{k, i}$ $(k=1,2, \ldots, L)$ are solved with Eq. (10) based on the following criterion (Shen et al. 2014):

$\min : \xi_{i}^{T} \boldsymbol{\Lambda}^{-1} \xi_{i}$,

where $\boldsymbol{\Lambda}$ is diagonal matrix of eigenvalues $\lambda_{k}$, which is the covariance matrix of PCs. The solution of Eq. (10) is as follows:

$\xi_{i}=\boldsymbol{\Lambda} \mathbf{G}_{i}^{T}\left(\mathbf{G}_{i}^{T} \boldsymbol{\Lambda} \boldsymbol{G}_{i}\right)^{-} \boldsymbol{y}_{i}$.

The symbol "-" denotes the pseudo-inverse of a matrix.

If the non-diagonal elements of $\mathbf{G}_{i}$ are all set to zero, Eq. (14) can be further simplified as

$$
\begin{gathered}
a_{k, i}=\frac{1}{1-\sum_{i+j-1 \in \bar{S}_{i}} v_{j, k}^{2}} \sum_{i+j-1 \in S_{i}} x_{i+j-1} v_{j, k} \\
1 \leq k \leq L, 1 \leq i \leq N-L+1 .
\end{gathered}
$$

Supposing that $v_{1, k}=v_{2, k}=\cdots=v_{L, k}=1 / \sqrt{L}$ at the missing data points, the solution of Eq. (15) will be reduced to Eq. (6). Therefore, the SSAM approach is a special case of our ISSA approach. The first several PCs contain most variance; the element $x_{i+j-1}$ can be approximately reproduced with the first several PCs in Eq. (8).

The main difference of our ISSA approach from the SSAM approach of Schoellhamer (2001) is in calculating the PCs. We produce the PCs from observed data with Eq. (14) according to the power spectrum (eigenvalues) and eigenvectors of the PCs, while Schoellhamer (2001) calculates the PCs from observed data with Eq. (6) only according to the eigenvectors and uses the scale factor $L / L_{i}$ to compensate the missing value. We have pointed out that this scale factor can be derived from Eq. (15), which is the simplified version of our ISSA approach, by supposing the missing data points with the same eigenvector elements. Therefore the performance of our ISSA approach is better than SSAM of Schoellhamer (2001). The only disadvantage of our method is that it will cost more computational effort.

\section{Performance of ISSA with synthetic time series}

The same synthetic time series as in Schoellhamer (2001) are used to analyse the performance of ISSA compared to SSAM. The synthetic SSC time series is expressed as

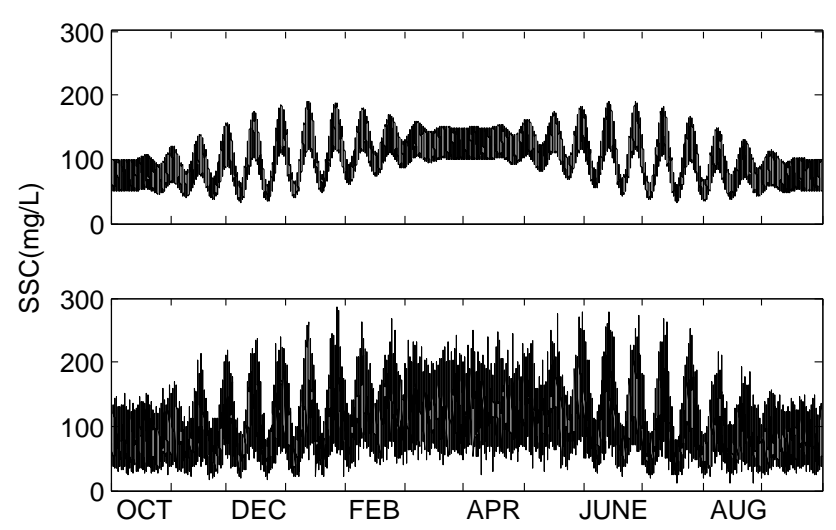

Figure 1. Periodic signal $c_{\mathrm{S}}(t)$ (top panel) and Synthetic time series (bottom panel).

$c(t)=0.2 R(t) c_{\mathrm{s}}(t)+c_{\mathrm{s}}(t)$,

where $R(t)$ is a time series of Gaussian white noise with zero mean and unit standard deviation; $c_{\mathrm{s}}(t)$ is the periodic signal expressed as

$$
\begin{aligned}
c_{\mathrm{s}}(t) & =100-25 \cos \omega_{\mathrm{s}} t+25\left(1-\cos 2 \omega_{\mathrm{s}} t\right) \sin \omega_{\mathrm{sn}} t \\
& +25\left(1+0.25\left(1-\cos 2 \omega_{\mathrm{s}} t\right) \sin \omega_{\mathrm{sn}} t\right) \sin \omega_{\mathrm{a}} t .
\end{aligned}
$$

The periodic signal oscillates about the mean value $100 \mathrm{mg} \mathrm{L}^{-1}$ including the signals with seasonal frequency $\omega_{\mathrm{s}}=2 \pi / 365 \mathrm{day}^{-1}$, spring/neap angular frequency $\omega_{\mathrm{sn}}=2 \pi / 14 \mathrm{day}^{-1}$ and advection angular frequency $\omega_{\mathrm{a}}=2 \pi / 12.5 / 24 \mathrm{day}^{-1}$. The 1 year of synthetic SSC time series $c(t)$, starting at 1 October with $15 \mathrm{~min}$ time step, is presented at the bottom of Fig. 1, the corresponding periodic signal $c_{\mathrm{s}}(t)$ is shown at the top of Fig. 1.

Although the selection of window length is an important issue for SSA (Hassani et al., 2012; Hassani and Mehmoudvand, 2013), this paper chooses the same window length ( $L=120)$ as that in Schoellhamer (2001) in order to compare the performance of the proposed method with that of Schoellhamer. Using the synthetic time series we compute the lagged correlation matrix and the variances of each mode. The first four modes contain the periodic components, which account for $72.3 \%$ of the total variance; in particular, the first mode contains $50.2 \%$ of the total variance. In order to evaluate the accuracies of reconstructed PCs from the time series with different percentages of missing data, following the approach of Shen et al. (2014), we compute the relative errors of the first four modes derived by ISSA and SSAM with the following expression:

$p=\frac{1}{N} \sum_{i=1}^{N} \sqrt{\frac{\left(\boldsymbol{a}_{i}-\boldsymbol{a}_{0}\right)^{T}\left(\boldsymbol{a}_{i}-\boldsymbol{a}_{0}\right)}{\boldsymbol{a}_{0}^{T} \boldsymbol{a}_{0}}} \times 100 \%$,

where the symbol " $T$ " denotes the transpose of a matrix, $p$ denotes relative error, $N$ is the number of repeated experiments, $\boldsymbol{a}_{i}$ is the reconstructed PCs of the $i$ th experiment 


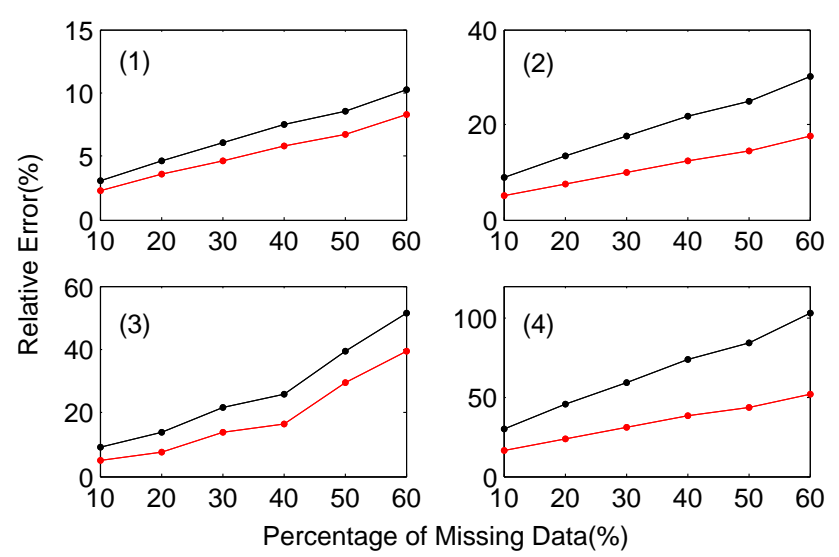

Figure 2. Relative errors of first four PCs (ISSA: red line; SSAM: black line).

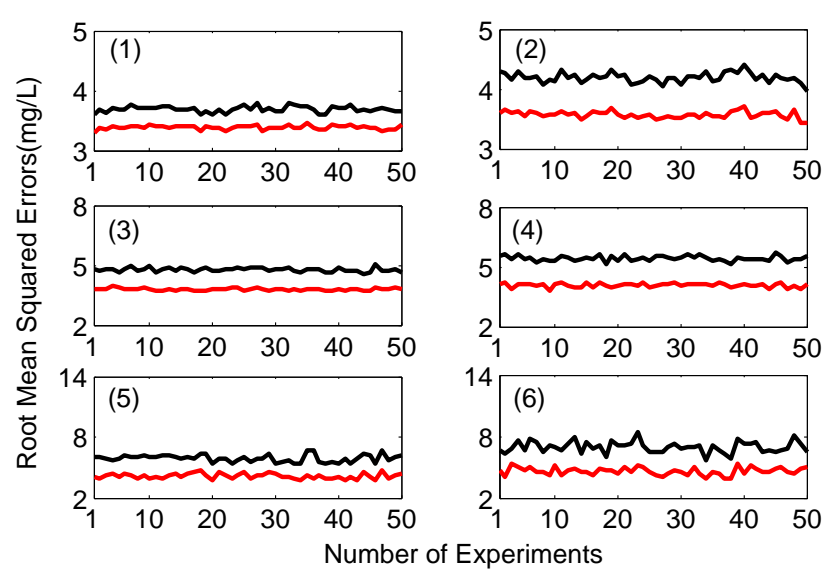

Figure 3. RMSE of 50 experiments, (1)-(6) represent percentage of missing data ranging from 10 to $60 \%$ in $10 \%$ increments.

from data missing time series, and $\boldsymbol{a}_{0}$ denotes the PCs reconstructed from the time series without missing data. We design the experiment of missing data by randomly deleting the data from the synthetic time series. The percentage of deleted data is from 10 to $60 \%$ with an increase of $10 \%$ each time. Then, we reconstruct the first four PCs from the data-deleted synthetic time series using both SSAM and ISSA, and repeat the experiments 50 times. The relative errors of the first four PCs are presented in Fig. 2, from which we clearly see that the accuracies of reconstructed PCs by our ISSA are obviously higher than those by SSAM, especially for the second and fourth PCs. In the case of $60 \%$ missing data, the accuracy improvements are up to $19.64,41.34,23.27$ and $50.30 \%$ for the first four PCs, respectively.

We reconstruct the time series $\hat{c}(t)$ using the first four PC modes and then evaluate the quality of reconstructed series by examining the error $\Delta \hat{c}(t)=\hat{c}(t)-c_{\mathrm{S}}(t)$. For the cases whose missing data are between 10 to $50 \%$ over the whole time series, the reconstructed component of the time series
Table 1. Mean absolute reconstruction error and mean root mean squared error of simulated time series with different percentage of missing data $\left(\mathrm{mg} \mathrm{L}^{-1}\right)$.

\begin{tabular}{|c|c|c|c|c|c|c|}
\hline \multirow{2}{*}{$\begin{array}{l}\text { Percentage } \\
\text { of missing } \\
\text { data }(\%)\end{array}$} & \multicolumn{3}{|c|}{ MARE } & \multicolumn{3}{|c|}{ MRMSE } \\
\hline & SSAM & ISSA & $\begin{array}{l}\text { IMP } \\
(\%)\end{array}$ & SSAM & ISSA & $\begin{array}{l}\text { IMP } \\
(\%)\end{array}$ \\
\hline 0 & 2.48 & 2.48 & 0 & 2.06 & 2.06 & $0 \%$ \\
\hline 10 & 2.87 & 2.60 & 9.41 & 3.68 & 3.38 & 2.21 \\
\hline 20 & 3.26 & 2.73 & 16.26 & 4.19 & 3.56 & 15.04 \\
\hline 30 & 3.71 & 2.90 & 21.83 & 4.76 & 3.78 & 20.59 \\
\hline 40 & 4.22 & 3.11 & 26.30 & 5.42 & 4.07 & 24.91 \\
\hline 50 & 4.57 & 3.17 & 30.63 & 5.89 & 4.14 & 29.71 \\
\hline 60 & 5.37 & 3.52 & 34.45 & 6.96 & 4.60 & 33.91 \\
\hline $\begin{array}{l}\text { SF Bay } \\
\text { example }\end{array}$ & 3.38 & 3.08 & 8.87 & 2.70 & 2.29 & 15.19 \\
\hline
\end{tabular}

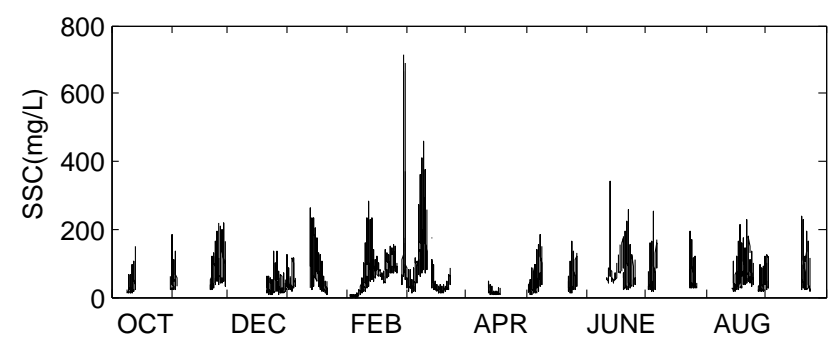

Figure 4. Mid-depth SSC time series at San Mateo Bridge during water year 1997.

is calculated only when the percentage of missing data in the window size is less than $50 \%$; while for the cases whose overall missing data already reach $60 \%, 60 \%$ missing data is allowed in the window size. In Fig. 3, we demonstrate the root mean squared errors (RMSEs) of each experiment of different percentages of missing data. The RMSE is computed with $\Delta \hat{c}(t)$ as

$\mathrm{RMSE}=\sqrt{\sum_{j=1}^{M} \Delta \hat{c}^{2}\left(t_{j}\right) / M}$

where $M$ is the number of data points involved in the experiment.

As can be seen from the Fig. 3, the RMSEs of ISSA are much smaller than those of SSAM for the same experiment scenarios. In Table 1, we present the mean absolute reconstruction error (MARE) and mean root mean squared error (MRMSE) of 50 experiments with different percentages of missing data.

Obviously, if there are no missing data, the ISSA coincides with SSAM. If the percentage of missing data increases, both MARE and MRMSE will become larger. In Table 1, all the MARE and MRMSE of ISSA are smaller than those of SSAM. When the percentage of missing data reaches $50 \%$, the MARE and MRMSE are 3.17 and $4.14 \mathrm{mg} \mathrm{L}^{-1}$ for ISSA, and 4.57 and $5.89 \mathrm{mg} \mathrm{L}^{-1}$ for SSAM, respectively. The im- 
Table 2. Maximum, minimum and mean absolute residuals of SSAM and ISSA.

\begin{tabular}{lrr}
\hline Residuals $\left(\mathrm{mg} \mathrm{L}^{-1}\right)$ & SSAM & ISSA \\
\hline Maximum & 145.05 & 126.61 \\
Minimum & -432.20 & -227.70 \\
Mean absolute residuals & 8.19 & 8.00 \\
SD & 13.48 & 12.27 \\
\hline
\end{tabular}

proved percentage (IMP) of ISSA with respect to SSAM is also listed in Table 1. As the amount of missing data increases, the IMPs of both MARE and MRMSE increase as well. Moreover, when the synthetic time series with the missing data is same as the real SSC time series of Fig. 4, the IMPs of MARE and MRMSE are 8.87 and $15.19 \%$, respectively.

\section{Performance of ISSA with real time series}

The mid-depth SSC time series at San Mateo Bridge is presented in Fig. 4, which contains about $61 \%$ missing data. This time series was reported by Buchanan and Schoellhamer (1999) and Buchanan and Ruhl (2000), and analysed by Schoellhamer (2001) using SSAM. We analyse this time series using our ISSA with the window size of $30 \mathrm{~h}(L=120)$ comparing with SSAM. The first 10 modes represent dominant periodic components as shown in Schoellhamer (2001) which contain $89.1 \%$ of the total variance. Therefore, we reconstruct the time series with the first 10 modes when the missing data in a window size is less than $50 \%$.

The residual time series, e.g. the differences of observed minus reconstructed data, are presented in Fig. 5. The maximum, minimum and mean absolute residuals as well as the SD are presented in Table 2. It is clear that both maximum and minimum residuals are significantly reduced by using ISSA approach. The SD of our ISSA is reduced by $8.6 \%$. The squared correlation coefficients between the observations and the reconstructed data from ISSA and SSAM are 0.9178 and 0.9046 , respectively, which reflect that the reconstructed time series with our ISSA can indeed, to very large extent, specify the real time series.

\section{Conclusions}

We have developed the ISSA approach in this paper for processing the incomplete time series by using the principle that a time series can be reproduced using its principal components. We prove that the SSAM developed by Schoellhamer (2001) is a special case of our ISSA. The performances of ISSA and SSAM are demonstrated with a synthetic time series, and the results show that the relative errors of the first four principal components by ISSA are sig-

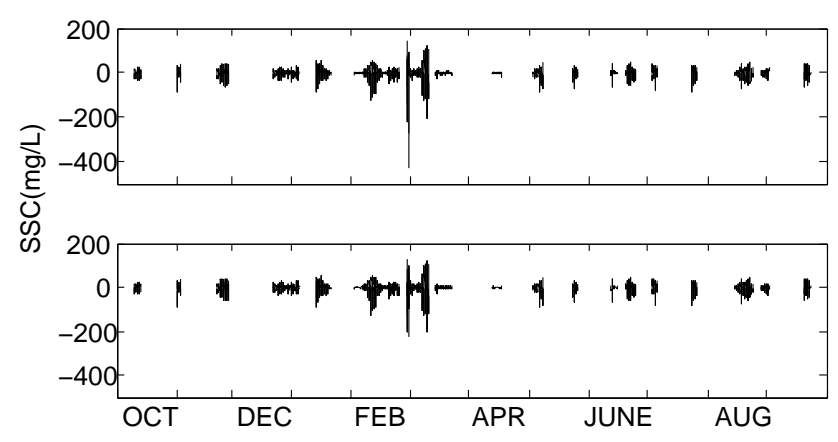

Figure 5. Residual series after removing reconstructed signals from the first 10 modes (top panel: SSAM; bottom panel: ISSA).

nificantly smaller than those by SSAM. As the fraction of missing data increases, the improvement of the relative error becomes greater. When the percentage of missing data reaches $60 \%$, the improvements of the first four principal components are up to $19.64,41.34,23.27$ and $50.30 \%$, respectively. Moreover, when the missing data account for $60 \%$, the MARE and MRMSE derived by ISSA are 3.52 and $4.60 \mathrm{mg} \mathrm{L}^{-1}$, and by SSAM are 5.37 and $6.96 \mathrm{mg} \mathrm{L}^{-1}$. The corresponding improvements of ISSA with respect to SSAM are 34.45 and $33.91 \%$. When the missing data of synthetic time series is the same as the real SSC time series, the improvements of MARE and MRMSE are 8.87 and $15.19 \%$, respectively. The SD derived from the real SSC time series at San Mateo Bridge by ISSA and SSAM are 12.27 and $13.48 \mathrm{mg} \mathrm{L}^{-1}$, and the squared correlation coefficients between the observations and the reconstructed data from ISSA and SSAM are 0.9178 and 0.9046 , respectively. Therefore, ISSA can indeed, to a great extent, retrieve the informative signals from the original incomplete time series.

\section{The Supplement related to this article is available online at doi:10.5194/npg-22-371-2015-supplement.}

Author contributions. Y. Shen proposed the improved singular spectrum analysis and F. Peng wrote the FORTRAN program and performed the simulations. Y. Shen, F. Peng and B. Li prepared the paper.

Acknowledgements. This work is sponsored by the Natural Science Foundation of China (Projects: 41274035, 41474017) and partly supported by State Key Laboratory of Geodesy and Earth's Dynamics (SKLGED2013-3-2-Z).

Edited by: I. Zaliapin

Reviewed by: two anonymous referees 


\section{References}

Broomhead, D. S. and King, G. P.: Extracting qualitative dynamics from experimental data, Physica D, 20, 217-236, 1986.

Buchanan, P. A. and Ruhl, C. A.: Summary of suspended-solids concentration data, San Francisco Bay, California, water year 1998, Open File Report 99-189, US Geological Survey, San Francisco, 41 pp., 2000.

Buchanan, P. A. and Schoellhamer, D. H.: Summary of suspended solids concentration data, San Francisco Bay, California, water year 1997, Open File Report 00-88, US Geological Survey, San Francisco, 52 pp., 1999.

Golyandina, N. and Osipov, E.: The "Catterpillar"-SSA method for analysis of time series with missing data, J. Stat. Plan. Inf., 137, 2642-2653, 2007.

Hassani, H. and Mahmoudvand, R.: Multivariate singular spectrum analysis: a general view and new vector forecasting approach, Int. J. Energy Stat., 1, 55-83, 2013.

Hassani, H., Mahmoudvand, R., Zokaei, M., and Ghodsi, M.: On the Separability between signal and noise in singular spectrum analysis, Fluct. Noise Lett., 11, 1-11, 2012..

Kondrashov, D. and Ghil, M.: Spatio-temporal filling of missing points in geophysical data sets, Nonlin. Processes Geophys., 13, 151-159, doi:10.5194/npg-13-151-2006, 2006.
Oropeza, V. and Sacchi, M.: Simultaneous seismic data denoising and reconstruction via multichannel singular spectrum analysis, Geophysics, 76, 25-32, 2011.

Robertson, A. W. and Mechoso, C. R.: Interannual and decadal cycles in river flows of southeastern South America, J. Climate, 11, 2570-2581, 1998.

Rodrigues, P. C. and de Carvalho, M.: Spectral modeling of time series with missing data, Appl. Math. Model., 37, 4676-4684, doi:10.1016/j.apm.2012.09.040, 2013.

Schoellhamer, D. H.: Singular spectrum analysis for time series with missing data, Geophys. Res. Lett., 28, 3187-3190, 2001.

Schoellhamer, D. H.: Variability of suspended-sediment concentration at tidal to annual time scales in San Francisco Bay, USA, Cont. Shelf Res., 22, 1857-1866, 2002.

Shen, Y., Li, W., Xu, G., and Li, B.: Spatiotemporal filtering of regional GNSS network's position time series with missing data using principal component analysis, J. Geodesy, 88, 1-12, doi:10.1007/s00190-013-0663-y, 2014.

Vautard, R., Yiou, P., and Ghil, M.: Singular-spectrum analysis: A toolkit for short, noisy, chaotic signals, Physica D, 58, 95-126, 1992.

Zotova, L. V. and Shum, C. K.: Multichannel singular spectrum analysis of the gravity field from grace satellites, AIP Conf. Proc., 1206, 473-479, 2010. 